\title{
Non-Axisymmetric Radiation from Surface-Wave Oscillator Driven by Weakly Relativistic Electron Beam*)
}

\author{
Yuta ANNAKA, Kazuo OGURA, Kosuke OZAWA, Makoto NAKASONE and Yuki SATO \\ Graduate School of Science and Technology, Niigata University, Niigata 950-2181, Japan
}

(Received 6 September 2018 / Accepted 24 November 2018)

\begin{abstract}
In this paper, non-axisymmetric radiation from a W-band surface wave oscillator (SWO) is observed. The SWO is based on a cylindrical corrugated waveguide with a periodic structure on its inner wall. This SWO is driven by electron beams with energy of less than $100 \mathrm{keV}$ and a current on the order of $100 \mathrm{~A}$. The waveguide has transverse magnetic (TM) and transverse electric (TE) modes as its axisymmetric normal modes. Nonaxisymmetric modes also appear as a hybrid of TM and TE modes and form surface waves near the periodic structure. The frequencies of the non-axisymmetric modes increase as the azimuthal mode number $m$ increases. By injecting an annular electron beam into the SWO, many non-axisymmetric modes with $m$ up to 30 are excited and intense radiation is generated at frequenies up to $140 \mathrm{GHz}$.
\end{abstract}

(C) 2019 The Japan Society of Plasma Science and Nuclear Fusion Research

Keywords: terahertz wave source, surface wave oscillator, corrugated waveguide, non-axisymmetric mode

DOI: $10.1585 /$ pfr.14.2406015

\section{Introduction}

Terahertz-wave sources are in demand for applications such as non-destructive inspection, wireless communication, radar systems, and plasma control. Slow-wave devices are among the most promising high-power sources of terahertz waves [1]. These devices are based on a periodic structure in which the kinetic energy of an electron beam is converted into electromagnetic (EM) wave energy. The surface-wave oscillator (SWO) is a slow-wave device utilizing a cylindrical corrugated waveguide [2-5]. This corrugated waveguide has two axisymmetric normal modes: transverse magnetic (TM) and transverse electric (TE). Non-axisymmetric normal modes are a hybrid of TM and TE modes and have the phase factor $\exp (\operatorname{im} \theta)$, where $m$ is the azimuthal mode number and $\theta$ is the azimuthal angle. Many non-axisymmetric modes with various $m$ values arise in a cylindrical corrugated waveguide. A corrugated waveguide has a periodic structure on its inner wall. This structure reduces a phase velocity of EM wave slower than that of light. Both axisymmetric and non-axisymmetric modes may become a slow wave. The intensity distribution of the slow wave is concentrated on the surface of the structure when the corrugated waveguide is oversized: the inner radius of the waveguide is several times larger than the wavelength of an EM wave in free space. Such wave is called a surface wave. By injecting an electron beam into the waveguide, axisymmetric and non-axisymmetric surface-wave modes can be excited to generate intense radiation [6]. When many non-axisymmetric modes exist close to each other, we have a serious problem, so called

author'se-mail: annaka@eng.niigata-u.ac.jp

*) This article is based on the presentation at the 12th International Conference on Open Magnetic Systems for Plasma Confinement (OS2018). mode competition problem, which degrade device performance $[7,8]$. In order to understand the multimode nature of SWO, the characteristics of emanating from many nonaxisymmetric modes should be investigated.

In this study, we examine the excitation of nonaxisymmetric surface-wave modes in a W-band SWO. The frequency of a non-axisymmetric mode increases with the increase in the mode number $m$. The upper cutoff frequency of the non-axisymmetric mode with $m=30$ is $140 \mathrm{GHz}$, whereas that of the axisymmetric $\mathrm{TM}_{01}$ mode is $100 \mathrm{GHz}$. Non-axisymmetric radiation with higher frequencies are observed using the detecting systems with high-pass filters at cutoff frequencies of 74, 116, and $137 \mathrm{GHz}$. An electron beam is generated by applying a voltage pulse lower than $100 \mathrm{kV}$ to a cold cathode, yielding a current on the order of $100 \mathrm{~A}$.

\section{Experimental Setup}

In our experimental setup, a disk-type cold cathode and a corrugated waveguide are placed in a vacuum chamber. By applying voltage to the cathode, an electron beam is generated and is injected axially into the corrugated waveguide. A schematic of the corrugated waveguide with an axially propagating electron beam is shown in Fig. 1. The corrugated waveguide is made of aluminum and has a rectangular periodic structure on its inner wall. The size parameters of the structure are an average radius $R_{0}=15.1 \mathrm{~mm}$, a corrugation amplitude $h=0.3 \mathrm{~mm}$, a periodic length $z_{0}=0.5 \mathrm{~mm}$, and a groove width $d=0.3 \mathrm{~mm}$. The beam limiter, with an inner diameter of $29.7 \mathrm{~mm}$, is connected in front of the corrugated waveguide and functions as an anode. The cold cathode is located $10 \mathrm{~mm}$ away 


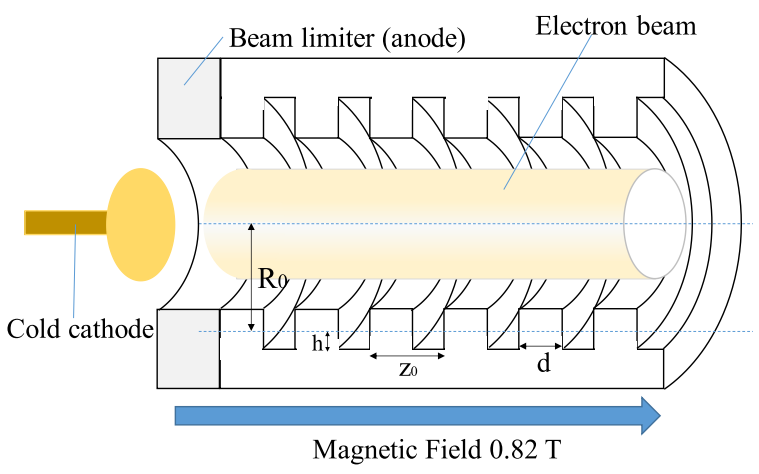

Fig. 1 Schematic of corrugated waveguide with beam diode and axially propagating annular electron beam. Beam diode is composed of cold cathode and beam limiter.

from the anode. This cathode is made of oxygen-free copper and its diameter is $29.0 \mathrm{~mm}$. In order to generate an annular electron beam, a dielectric fiber is attached to the edge of the cathode surface $[9,10]$. The pulse-forming line is connected to the cathode and the voltage pulse up to $100 \mathrm{kV}$ is applied to the cathode. A $0.82 \mathrm{~T}$ uniform magnetic field is generated by 10 solenoid coils. A beam current is measured by a Rogowski coil.

Intense radiation is generated while the electron beam propagates within the corrugated waveguide. The radiation passes to the outside of the vacuum chamber through an output window and is observed by F-band, G-band and Y-band detecting systems. F-band, G-band and Y-band are EIA standard. The detecting system is composed of a horn antenna, a rectangular waveguide, a directional coupler, and a crystal diode detector. The rectangular waveguides have cutoff frequencies and serve as high-pass filters. The cutoff frequencies are $74 \mathrm{GHz}$ for the F-band, $116 \mathrm{GHz}$ for the G-band, and $137 \mathrm{GHz}$ for the Y-band. The directional coupler is used as a 20-dB attenuator in the F-band system although no directional coupler is used in the G-band and Y-band systems. The diode detectors are ELVA-1 ZBD08 for the F-band, Pacific Millimeter Product GD for the G-band and ELVA-1 ZBD-06 for the Y-band. The output voltages of the ELVA-1 ZBD-08 and ZBD-06 detectors are calibrated to the absolute values of power using a Gunn oscillator at $100 \mathrm{GHz}$. The output voltage of the G-band detector is calibrated to the absolute value of power by a G-band BWO [11]. The detecting systems are located $600 \mathrm{~mm}$ away from the window.

\section{Non-Axisymmetric Mode}

Non-axisymmetric modes are a hybrid of TM and TE modes and are commonly designated by the letters EH and HE. The definitions of EH and HE are arbitrary. In this study, we use the definition common in plasma physics research: The TM mode is dominant in The EH mode and the TE mode is dominant in the HE mode [12]. Using numerical code developed in Ref. [13], the dispersion curves

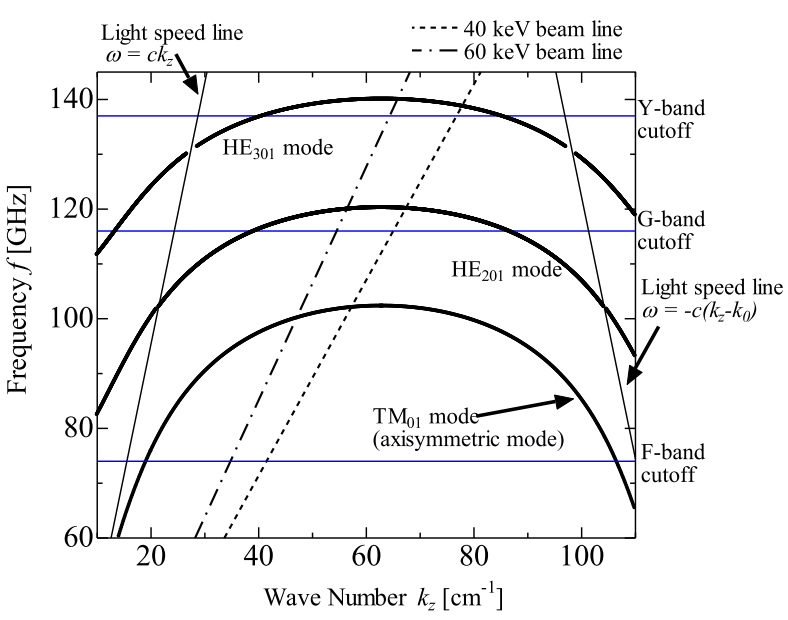

Fig. 2 Dispersion curves of axisymmetric $\mathrm{TM}_{01}$ mode and nonaxisymmetric $\mathrm{HE}_{201}$ and $\mathrm{HE}_{301}$ modes.

of the waveguide modes are calculated from the size parameters and are shown in Fig. 2. In this figure, the vertical axis represents the frequency $f$ and the horizontal axis represents the wavenumber $k_{z}$. The dispersion curves indicate the axisymmetric $\mathrm{TM}_{01}$ mode and the non-axisymmetric $\mathrm{HE}_{\mathrm{m} 1}$ modes with $m=20$ and 30. The upper cutoff frequencies of the modes appear at $k_{z}=62.83 \mathrm{~cm}^{-1}$ and are $100 \mathrm{GHz}$ for $m=0,120 \mathrm{GHz}$ for $m=20$, and $140 \mathrm{GHz}$ for $m=30$. The thin solid lines plot the light lines for $\omega=c k_{z}$ and $\omega=-c\left(k_{z}-2 \pi / z_{0}\right)$, where $\omega=2 \pi f$ is the angular frequency and $c$ is the velocity of light. The phase velocities of the non-axisymmetric modes $\omega / k_{z}$ are slower than $c$, so these HE modes are slow waves. As mentioned in Sec. 1, slow waves are surface waves on the vicinity of the periodic structure. The dashed and dash-dotted lines indicate the 40 and $60 \mathrm{keV}$ beam lines $\omega=v k_{z}$, respectively, where $v$ is the beam velocity. The electron beam interacts with a non-axisymmetric mode at the intersection of the beam line and dispersion curve, leading to intense radiation. The electron beam in an oversized corrugated waveguide interacts with the TM modes, but not with the TE modes. The TM mode becomes dominant in the $\mathrm{HE}_{\mathrm{m} 1}$ mode in the slow-wave region in which the phase velocity is slower than $c$ and an electron beam interacts with $\mathrm{HE}_{\mathrm{m} 1}$ modes, whereas the TE mode is dominant in HE mode around $k_{z}=0$ [14]. No EH modes become surface waves at frequencies below $150 \mathrm{GHz}$, so these may not contribute to radiation. The blue solid lines indicate the cutoff frequencies of the detecting systems that were discussed in Sec. 2. The upper cutoff the frequencies of $\mathrm{HE}_{\mathrm{m} 1}$ modes are plotted as a function of the mode number $m$ in Fig. 3. The frequencies of $\mathrm{HE}_{\mathrm{m} 1}$ modes increase as the mode number $m$ increases. Radiation may be observed when the frequency is sufficiently larger than the cutoff frequencies of the detecting systems. The G- and Y-band detecting systems are expected to observe radiations with mode number $m$ larger than 20 and 30, respectively. 


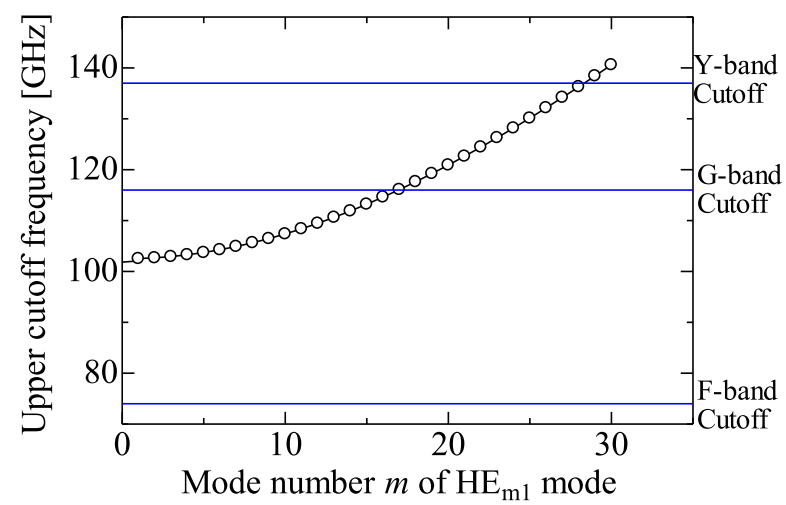

Fig. 3 Dependence of upper cutoff frequency of $\mathrm{HE}_{\mathrm{m} 1}$ mode on mode number $m$.

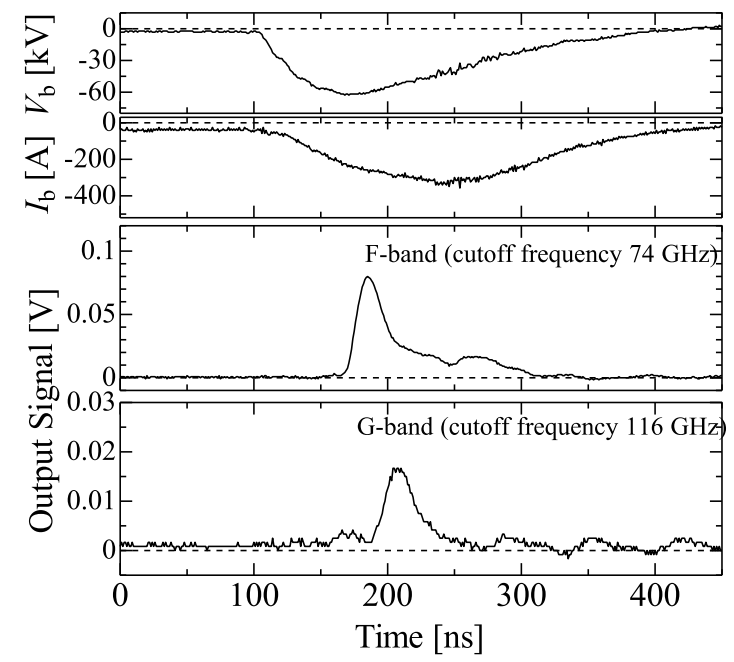

Fig. 4 Temporal evolution of cathode voltage, beam current, and F- and G-band signals.

\section{Experimental Result}

With an electron beam injected into the corrugated waveguide, the time evolution of the cathode voltage, the beam current, and the output signals of the F-band and Gband detectors are obtained as shown in Fig. 4. The F-band signal is decreased by a $20-\mathrm{dB}$ attenuator. The peak of Fband signal is observed at $185 \mathrm{~ns}$ where the cathode voltage is $79 \mathrm{kV}$ and beam current $257 \mathrm{~A}$. The observed F-band peak power is $760 \mathrm{~mW}$. The peak of the G-band signal is observed at $210 \mathrm{~ns}$ where the cathode voltage is $68 \mathrm{kV}$ and the current is $290 \mathrm{~A}$. The observed peak of the G-band power is $166 \mathrm{~mW}$. Signals from the G- and Y-band detecting systems are shown in Fig. 5. Their maximum values appear at the same time at $163 \mathrm{~ns}$, when the cathode voltage and current are $65 \mathrm{kV}$ and $224 \mathrm{~A}$, respectively. The observed power at $163 \mathrm{~ns}$ is $749 \mathrm{~mW}$ for the G-band and $16 \mathrm{~mW}$ for the Y-band. Considering the cutoff frequencies of the G- and Y-band systems, the frequencies are over $116 \mathrm{GHz}$ for G-band radiation and $137 \mathrm{GHz}$ for Y-band radiation. The second peak in the G-band signal appears

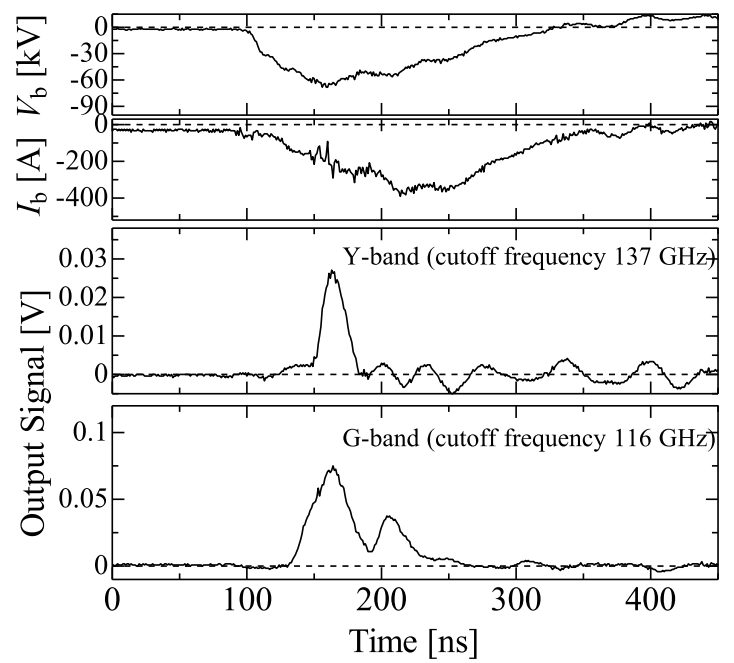

Fig. 5 Temporal evolution of cathode voltage, beam current, Yand G-band signals.

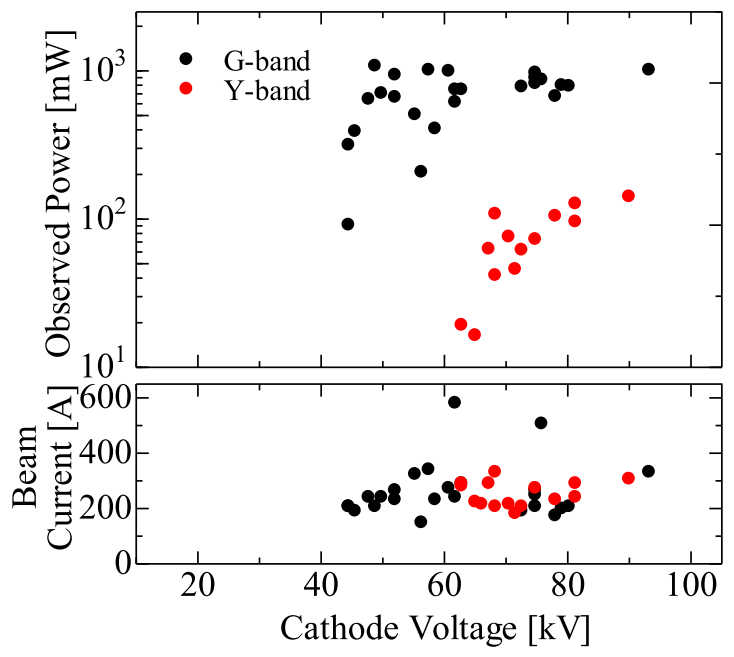

Fig. 6 Dependence of G- and Y-band observed power and beam current on cathode voltage.

at $204 \mathrm{~ns}$, at which point the cathode voltage and current are $55 \mathrm{kV}$ and $299 \mathrm{~A}$, respectively. The G-band power at $204 \mathrm{~ns}$ is $374 \mathrm{~mW}$. No meaningful Y-band signal is observed around $204 \mathrm{~ns}$. In accordance with the cutoff frequencies, the frequency of the G-band radiation lies in the range from 116 to $137 \mathrm{GHz}$. By varying the cathode voltage, the peak values of the G- and Y-band signals and the beam current at the corresponding time are plotted as the function of the cathode voltage in Fig. 6. In order to collect the data, over 50 shots are observed. The beam currents are distributed between 100 and $600 \mathrm{~A}$. G-band radiation is detected in the voltage region above $40 \mathrm{kV}$. The maximum observed power is on the order of $1 \mathrm{~W}$. On the other hand, Y-band radiation is observed in the voltage range above $60 \mathrm{kV}$. The maximum observed power is on the order of $0.1 \mathrm{~W}$. 
Intense radiation is observed by G- and Y-band detecting systems with cutoff frequencies of 116 and $137 \mathrm{GHz}$, respectively. G-band radiation is observed with cathode voltages above $40 \mathrm{kV}$, and Y-band radiation is observed with cathode voltages above $60 \mathrm{kV}$. The G-band power level is 10 times higher than the Y-band. As the data in Figs. 2 and 3 show, the G-band signals in Figs. 4, 5, and 6 correspond to the excitation of $\mathrm{HE}_{\mathrm{m} 1}$ modes with $m$ above 20. Likewise, the Y-band signals in Figs. 5 and 6 may result from the excitation of $\mathrm{HE}_{\mathrm{m} 1}$ modes with mode numbers $m$ above 30 .

These experimental data show that many $\mathrm{HE}_{\mathrm{m} 1}$ modes are excited, with $m$ up to at least 30. By comparing the power levels of the G- and Y-band signals, the intensity of non-axisymmetric radiation appears to decrease as the mode number $m$ increases from 20 to 30 . Hence, the radiation with the lowest azimuthal mode number may be dominant in these signals. In other words, radiation with $m=20$ and 30 seems to be dominant in the G- and Yband signals, respectively. As Fig. 6 shows, G- and Y-band radiation is detected with cathode voltages above 40 and $60 \mathrm{kV}$, respectively. This result suggests that a threshold cathode voltage for non-axisymmetric radiation exists and that this threshold value increases as the mode number $m$ increases. In Fig. 5, the Y-band signal disappears around 204 ns, although a G-band signal is observed. At this moment, the cathode voltage is $55 \mathrm{kV}$ and the signal is over the threshold value for $m=20$ but below the threshold for $m=30$.

\section{Discussion and Conclusion}

F-band signals are observed from around $10 \mathrm{kV}$ and correspond to the W-band oversized SWO oscillation presented in Ref. [2]. This oversized SWO has been shown to be a multimode system through radiation pattern measurements. We observed the excitation of many $\mathrm{HE}_{\mathrm{m} 1}$ modes up to $m=30$ on the basis of the difference among their frequencies. The multimode nature of our oversized SWO leads to the existence of many non-axisymmetric modes. In Fig. 4, the F-band power level is about 4.6 times greater than the G-band power level. This difference results from the fact that the F-band signal may include radiation in the $\mathrm{TM}_{01}$ and $\mathrm{HE}_{\mathrm{m} 1}$ modes with mode numbers $m$ greater than 1. Although the F-band detector is sensitive enough to detect radiation up to $m=30$, the main contribution to nonaxisymmetric modes comes from radiation with $m$ much less than 20 , which exist very closely to the $\mathrm{TM}_{01}$ mode.

In conclusion, our experiment returned novel data about non-axisymmetric radiation from a W-band SWO. Non-axisymmetric $\mathrm{HE}_{\mathrm{m} 1}$ modes with a range of azimuthal mode numbers $m$ form surface waves on the surface of the SWO's periodic structure. When an annular electron beam is injected into the SWO, many $\mathrm{HE}_{\mathrm{m} 1}$ modes are excited. Intense radiation due to the excitation of non-axisymmetric $\mathrm{HE}_{\mathrm{m} 1}$ modes with azimuthal mode numbers up to $m=30$ are observed. We conclude that non-axisymmetric radiation is generated when the cathode voltage is over a certain threshold. This threshold value increases as the mode number $m$ increases. The results presented above contribute to our understanding of the multimode nature of SWO and to solving the mode competition problem. This solution will have an important effect on the development of terahertzwave sources.

[1] J.H. Booske, Phys. Plasmas 15, 055502 (2008).

[2] Min Thu San et al., Plasma Fusion Res. 11, 2406085 (2016).

[3] N.S. Ginzburg et al., Phys. Rev. Lett. 117, 204801 (2016).

[4] Min Thu San et al., IEEE Trans. Plasma Sci. 45, 30 (2017).

[5] J. Wang et al., Sci. Rep. 8, 6978 (2018).

[6] K. Ogura et al., J. Plasma Fusion Res. SERIES 6, 703 (2004).

[7] K. Mizuno et al., IEEE Trans. Electron Devices 20, 749 (1973).

[8] T.M. Antonsen, Jr. and B. Levush, Phys. Rev. Lett. 62, 1488 (1989).

[9] H. Oe et al., J. Plasma Fusion Res. SERIES 8, 1477 (2009).

[10] K. Yambe et al., IEEE Trans. Plasma Sci. 41, 2781 (2013).

[11] S. Magori et al., Plasma Fusion Res. 9, 3406032 (2014).

[12] O. Watanabe et al., Phys. Rev. E 63, 056503 (2001).

[13] Y. Takashima et al., J. Plasma Fusion Res. SERIES 8, 1512 (2009).

[14] H. Yamazaki et al., J. Plasma Phys. 72, 915 (2006). 